\title{
Creativity Is Connecting Things: The Role of Network Topology in Fostering Collective Creativity in Multi-Participant Asynchronous Online Discussions
}

\author{
Noa sher \\ University of Haifa \\ nsher01@campus.haifa.ac.il
}

\author{
Carmel Kent \\ University College London \\ kent.carmel@gmail.com
}

\author{
Sheizf Rafaeli \\ University of Haifa \\ sheizaf@univ.haifa.ac.i1
}

\begin{abstract}
Creativity derives from the ability to form new meaningful combinations out of available resources. Collective creativity is the product of a collaborative process, consisting of multiple interactions between group members and the shared content, which lead to the emergence of novel shared meanings. This exploratory research addresses the expression of collective creativity in multi-participant asynchronous online discussions, by proposing interactivity and emergence as key features of the collaborative creative process. The ability to connect posts in a non-sequential manner ("cross-linking") is suggested as the basis for the formation of emergent community-structures within the content, which reflect collectively constructed novel combinations. Initial indications for this process are presented by applying a combination of network analysis and qualitative inquiry to data from a multiparticipant virtual discussion, held as part of an online academic course. A methodology for extracting emergent themes is described.
\end{abstract}

\section{Introduction}

Platforms for online collaboration allow large groups of people to communicate and participate in ongoing, a-synchronic deliberations. They have become a prominent space for exchanging thoughts and ideas, sharing information and insights and generating collective knowledge, for professionals, laypeople and learners of all sorts [1, 2]. These distributed communities produce collective content products, whether as an intentional process or as a by-product of the multiple interactions between people and content.

The nature of the online discussion environment offers possible advantages for group-level creativity, as some of the effects of co-located groups, including productive blocking and groupthink may be reduced [3]. Asynchronous settings allow not only more time, but also the coexistence of several parallel discussions, that might feed one another, possibly raising the opportunity for novel ideas to form [4]. However, the theoretical possibility of a multi-dimensional largescale discussion that is productive and synergetic, is also constrained by the affordances of the platform [5].

In the work presented here, we reflect on collective creativity within large-group online collaborative discussions. We focus on interaction and emergence as key factors in the collective creative process. The work addresses the questions of whether and how interactivity and emergence, as indications of collective creativity, can be inferred from the network structure of a discussion composed of posts and links. To explore these questions, a large-group academic discussion, engaging nearly 150 participants, was examined. Through a combination of network analyses of the postnetwork and qualitative inquiry, we offer initial insight towards the contribution of linking posts within an online multi-participant discussion to collective creativity. This work attempts to: 1) further develop the operationalization of interactivity in online discussions based on network measures [6]; 2) build on insights from previous theoretical $[5,7]$ and qualitative [8] work to form an operationalization strategy for recognizing emergence of novel ideas in an online discussion, based on network structures formed by participants' linking of posts; and 3) combine the two for a better understanding of the process of collective creativity in multiparticipant online discussions.

\section{Theoretical background}

Creativity has long been considered a peak of human capabilities, at both the individual and group levels. While individual creativity refers to a new mental combination that is expressed in the world, group creativity refers to a product that is created through interaction by a group, a work team, or an ensemble [3].

$\mathrm{Yu}$, Nickerson, \& Sakamoto defined a collective creativity system as one in which crowds engage in nonroutine tasks through which novel output emerges [5]. They emphasize the difference between mere 
aggregation of the collective products and the combination of these products in a manner that produces novelty and suggest emergence as the principal mark of a collective creative process. Heylighen, Heath, and Van highlighted the role of recurrent, non-linear interactions in the emergence of novel knowledge through selforganization, and addressed the product of such processes as collective "mental content" [7]. The current work attempts to operationalize these concepts within networked online discussions, by searching for indications of emergence resulting from participants' interactive liking. The following sections elaborate on the manifestations of interactivity and emergence in online discussions, and on their roles in collective creativity.

\subsection{Interactivity as a basis for a collective creative process}

Within appropriate settings, group creativity benefits from the interaction of group members with the ideas and inputs of others [9]. Rafaeli and Sudweeks emphasized interactivity in online group communication as the key component in the process of producing shared meanings [10]. Interaction, consisting of recurrent relation to previously posted content, recursive updating, and shaping of the shared content, and continuous generation of shared meanings, serves as the basis for the gradual collective creation of something new [8]. Therefore, Interaction between people and content serves in this work as the required condition for a collective process to be considered.

\subsection{Emergent network structures as representations of creativity}

Interactivity is key in any collaborative process. However, for collaboration to induce group-level creativity, the settings must support the emergence of new meanings, concepts or ideas through the interactive process [11]. The form which emergence in online collaboration takes on can vary, and an operational definition is needed. One of the more operational definitions for emergence as a phenomenon, in general, is the appearance of system-level changes resulting from element-level activity, without top-down direction [12]. To better understand the ways this can be expressed in a collaborative setting, we turn to the concept of creativity within individuals. In individuals, it appears that the "element-level activity" might be the formation of connections or links between distant areas of the conceptual network, as opposed to linear advancements within a hierarchical, rigid "chain of thought". These contribute to reorganizations of the cognitive network, facilitating the formation of non-trivial associations, which translate into insight [13, 14]. Moving from the individual to the group level raises the question of the space in which such associations might occur. Stahl has suggested that group discourse in online collaboration platforms can be viewed as a medium for group cognitive processes [15]. Building on Stahl's idea that in such platforms the discourse itself is the substrate for the collaborative formation of new meanings [16], we focus the search for emergence on the collective discussion as both a process and a product. Accordingly, the current work is concerned with emergence within the discussion content, rather than among its participants. In other words, emergence is defined here as the formation of new ideas and concepts through associations and combinations of content, and not through associations and combinations of people (such as social cliques). This formation is expressed in changes to the structure of the network of posts that compose the discussion

Emergence within the collection of posts requires that they are organized in a manner that enables changes in the network structure through bottom-up activity. This points out a major constraint on emergence within traditional threaded discussions. This work addresses the unique feature that is required for alteration of the underlying topology of a discussion, a necessary condition for emergence and hence for creativity.

\subsection{Using emergent community structures for making sense of large-scale discussions}

One of the challenges brought about by multiparticipant online discussions is keeping track and making sense of the entire conversation [17]. While some studies have addressed this issue from the individual participant's perspective, the challenges apply at the collective level as well: how can the collective product of a multi-participant discussion be preserved and communicated as a whole, maintaining at least some of its complexity? Some approaches, such as Topic Modelling rely on text analysis to identify similarities and co-occurrence of phrases and extract prominent themes from the corpus of posts, with or without human moderation. These themes can be used for generating reduced networks that offer a simplified display of the major topics discussed, and the general relations between them [18]. However, methods relying on text analysis fall short in several ways: first, they only apply to text, and cannot be used for integrating other types of content in the network. Second, linking based on identical or similar textual expressions may miss more complex forms of expression, such as metaphors or analogies. This might be especially relevant to more distant connections, that might foster a higher level of abstraction [19]. Third, and perhaps most importantly, extracting implicit connections based on text rather than 
on intentional links created by participants means forgoing a substantial part of the knowledge held by the participants: the knowledge that is in the connections.

The goal of this work is to explore how interaction and emergence, as the theoretical constructs at the base of collective creativity, are reflected in the network of posts produced through a collaborative multi-participant online discussion. Interaction is regarded as a basis for collaboration and for the creation of a collective product. The interactivity of the discussion is examined based on the activity of participants in relation to content contributed by their peers. Emergence is operationalized as macro-level changes to the collective conceptualassociative network created through the discussion, deriving from the combined actions of participants. By examining the expression of these qualities in a multiparticipant, unmoderated, asynchronous discussion, we seek to better understand whether such an environment can harbor the conditions for collective creativity to take place. Additionally, we present the methodology that was used for extracting emergent themes from a networked discussion, based on links by participants.

\section{Affordances for collective creativity}

This work points to the combination of interaction and emergence as crucial features of a collaborative online discussion that can foster collective creativity. The following section introduces some of the affordances of platforms that enable and promote both.

\subsection{Sequential linking as the basis for interactivity}

Interactivity may be conceptualized as a process of relating to each other's postings by taking conversational turns [10]. The online environment enables several layers of interaction: among participants, between participants and content, and across content items. The latter occurs through participants' active effort to integrate new input into the network, by relating their posts to the existing network in a meaningful way. This iterative process of adding new content in a manner that relates to previous content is an essential part of collaborative knowledge construction [20]. Accordingly, for a platform to support interactivity on all levels, it must encourage adding new content within the context of existing content. This can be achieved in any environment that supports a "post and respond" format but is enhanced in a platform that requires posts to be linked to previous posts [21].

Inclusion of external links creates an even broader context for the discussion and enhances the basis for the construction of new knowledge and understanding [22].
External links should be regarded as an integral part of a discussion, as their amount and scope directly affect the richness and sophistication of the discussion.

\section{2 "Cross-linking" as the basis for emergence}

Online threaded discussions, characterized by a chronologically hierarchical structure, are still prominent in online collaborative environments [17, 23]. The threaded structure enables a linear display that facilitates "top-down" navigation. The discourse is commonly organized by discrete topics, which are either set in advance, established by a moderator or initiated during the course of the discussion by participants [24]. This format imposes a rigid structure, which hinders the discussion's potential to converge or self-organize in a meaningful way [25]. This structure supports a hierarchical associative sequence, that may promote an in-depth exploration of an idea or concept, but is limited to a linear progression and complicates forming interdomain connections. In their study of the collaboration on the Polymath Project, Kittur and Cranshaw noted that the linear, threaded nature of the environment posed a constraint on the integration of what ended up remaining several distinct separate discussions [4].

In contrast to threaded discussions, which are characterized by a tree-shaped topology, online collaboration platforms can be designed in a manner that allows networked topologies to emerge. A networktopology of a discussion represents a non-linear, less hierarchical organization of knowledge. The network structure itself is more dynamic and less constraining and can describe more complex relations between parts of the discussion. It holds possibilities for selforganization, which make it better suited for promoting emergent processes and novel formations. It has also been found to increase interactivity [21].

We use the term cross-links to refer to the feature that differentiates environments that support networked topologies from environments that can only generate tree-shaped dendrograms. Cross-links are links between existing posts that can be added at any point in the discussion. The connections formed by these links deviate from the sequential hierarchy and so break the rigid tree-shaped structure. Cross-links can reshape the discussion's structure by connecting separate "branches". Cross-linking may take on different forms such as hyperlinking, cross-referencing, tagging or even direct linking within a network display. While differing in many aspects, they all allow participants to point out relations and connections between different conceptual units. Cross-linking may represent a wide range of relationships between units of information. Links may imply a commonality, a contrast, a relation of induction or deduction, a shared domain or any other type of 
relation, so long as the connection between the two units provides meaning. This type of liking can be regarded as analogous to the forming of new links between mental representation of concepts within an individual, which have been suggested as the basis for creative insight [13]. Importantly, to enable a group-level process, these connections must be made public and be present at the collective level [8].

Figure 1 presents the difference in the effects of sequential vs. cross-linking on network topology.

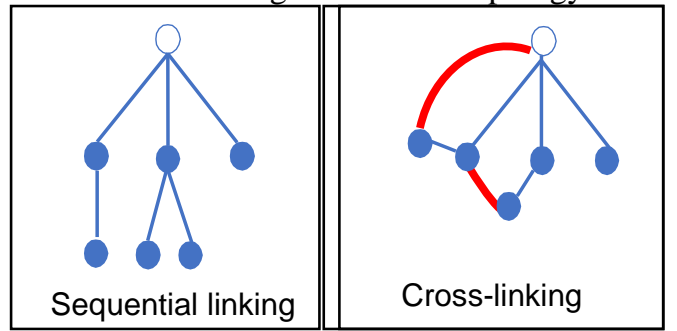

Figure 1. Cross-linking affects network structure

\subsection{Cross-linking's contribution to forming an emergent collective associative network}

On top of their 'traditional' roles in the formation of a collective knowledge-base which include adding new content, voting and viewing content by others, crosslinking affords participants the opportunity to play an active role in shaping the conversation. They can contribute their own insight to transform the structure of the network of connections at the collective level. The result is a structure that represents the collective conceptual map, created via the collaborative process. From a network-topology perspective, cross-linking can create "communities": densely connected modules which gather together posts relating to a concept [26]. This is a self-organizing process, independent of predetermined categorization. Links can also be formed across modules if participants recognize a connection between content units that were originally further apart. If these links remain sparse, they might function as "bridges" between different ideas. If the bridging links become denser, then the modules may merge to create a bigger overarching module. The process described here provides insight towards the way creativity can be afforded by cross-linking: it enables element-level activity to produce macro-level changes and allows new meanings, ideas and themes to emerge through bottomup combinations.

\section{Materials and data}

The methods section includes the description of a discussion platform that supports cross-linking by embedding a post-connecting feature within a network visualization of the discussion. While it makes crosslinking particularly straightforward, other forms of cross-linking, such as hyperlinking or tagging, also enable the extraction of collaboratively formed networks [27, 28]. Importantly, this work is concerned with environments in which the discussion itself is both the process and the product of collaboration. In environments that separate between process and product, such as wikis, creativity might be expressed in other fashions.

\subsection{The Ligilo discussion platform}

Ligilo is a hyperlinked discussion platform where each post is expressed as a node in a semantic network of posts. Using Ligilo, communities can create collective concept maps through online discussions. Posts can include text, multimedia and external links. A new post cannot stand alone, but rather has to be linked to a prior content post. It is similar to a standard discussion forum but has several distinctive features, including a visual display of the network of posts [20].

Critically, the visual network display includes a feature which allows participants to cross-link, by connecting any two existing posts they see as related, at any time. Adding such links induces two changes: first, it reshapes the network display of the posts, to include the new link and "pull" the newly connected nodes closer together (as well as nodes previously connected to them). This forms new structures that can be seen visually. Second, as in the case of sequential linking within the platform, after the connection is made the posts become "gateways" for each other. While navigating in a discussion-like view of the network, opening a post for reading directs the participant to all other posts connected to it. Accordingly, the new link affects the flow of post reading for subsequent readers

Figure 2 displays a screenshot of a post within the network-view display in Ligilo.

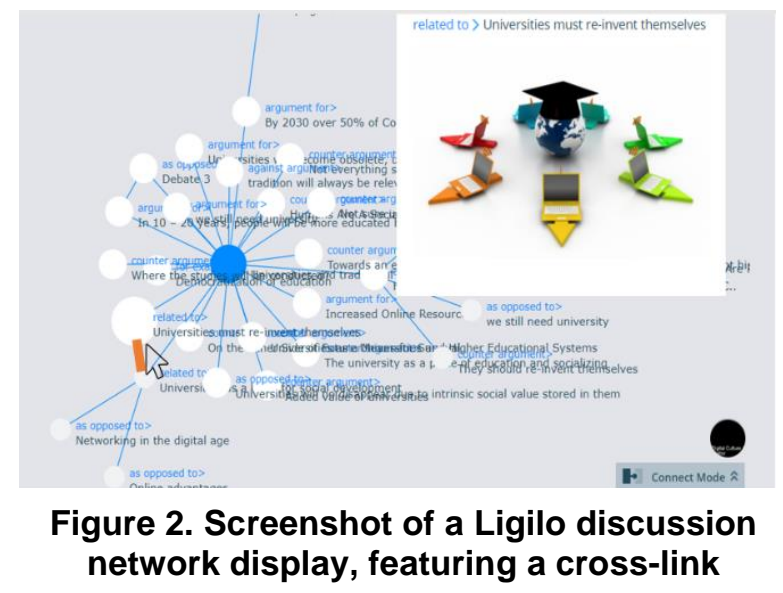


The work presented here is based on data collected from one Ligilo discussion scene. Ligilo has so far been used in multiple settings, mostly within academic environments. This particular discussion was chosen due to its scope, which included 145 active participants, comprising 143 students and 3 staff members, who produced a total of 821 posts. The discussion and its context will be described in detail in the next section.

\subsection{The collaborative discussion data}

The data we used was extracted from an online multi-participant asynchronous discussion, held as part of the requirements of an online course offered to graduate students. The 15-week course was hosted on the edX.org platform, through which the students were directed to their discussion arena on Ligilo. The discussion was held in the format of four open debates, each opened with a proposition about life in the Digital Era (e.g. "Control must always remain in human hands", "Traditional universities will become irrelevant").

4.1.1 Participant composition. 142 Graduate students participated in the discussion. Of these, 138 participants made some contribution other than a self-introduction post. Three more viewed posts other than selfintroductions by others, but did not post on their own, totaling in 141 student-participants engaging with the core of the discussion, and three staff members that were not active in the discussion after its initiation. The participants were Business Management students, Human Resources students and Information and Knowledge Management students, who were joined by several students from other departments. The discussion also included the four initial debate propositions, two examples and one additional post by a staff member.

4.1.2 The assignment. Participants were required to contribute at least three posts: one supportive argument, one contradictive argument, and one rebuttal post - a reaction to a fellow participant. While this was the required minimum, participants were encouraged to further engage in the discussion. They were notified that grading will consider both the quality of the arguments based on logical structure, grounded claims and linking to external references, and the overall contribution to the advancement of the discussion. The latter was intentionally vague, giving the participants leeway to interpret what such a contribution may be. They were encouraged to back their arguments with references to external resources. Participants were notified about the connect-mode feature but were not required to use it.

4.1.3 Extent of participation. About half of the participants (74) posted more than the required minimum (three posts). 15 participants (about 10\%) contributed more than 9 posts, and one participant contributed 22 posts. $14 \%$ of the participants engaged in cross-linking by using the connect-mode feature.

4.1.4 The network of posts. The network of posts was reconstructed based on the Ligilo network data, using the $\mathrm{R}$ igraph package [29]. The network comprised of all posts and links that were part of the main discussion, after removing a thread dedicated to self-introduction and a mini-thread that featured technical questions and answers, which were not an integral part of the discussion. Posts that were removed from the discussion by their creators ( $5 \%$ of posts) were not included in the data, as they were not part of the final graph. Table 1 summarizes the final graph used in the analysis.

\section{Table 1. The final graph stats}

\begin{tabular}{ll}
\hline Participants & 141 \\
\hline Nodes (posts) & 673 \\
\hline Links & 750 \\
\hline Percent of cross-links & $10 \%$ \\
\hline $\begin{array}{l}\text { Percent of cross-link } \\
\text { contributors }\end{array}$ & $14 \%$ \\
\hline Timespan & 101 days \\
\hline
\end{tabular}

Although the process of adding posts is directed, as each new post follows an existing one, the post-graph was created as an undirected graph, for two reasons:

1. Once posted, navigation along graph posts through their links is bi-directional. Participants can move from a post to any other post connected to it, regardless of the original direction of the link.

2. On a theoretical level, the association between ideas is not a one-way street. The current research views the discussion as a holistic product, which is why we are concerned with the eventual network of connections between all posts.

Figure 3 presents the full discussion graph

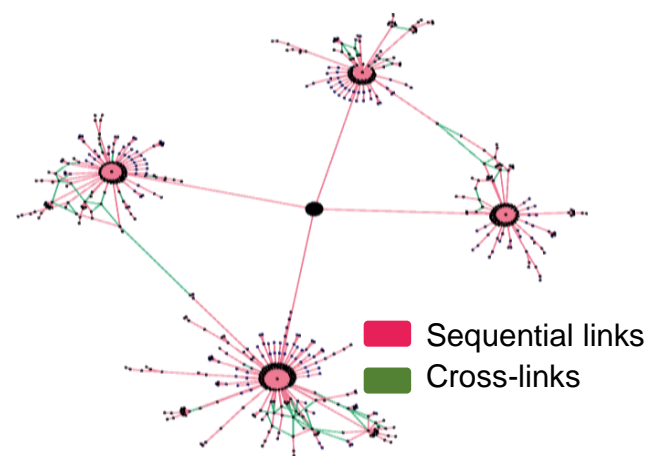

Figure 3. The graph of posts and links 


\section{Analyses and findings}

\subsection{Establishing interactivity}

Interaction has a key role in the collective process and differentiates collective work from a mere aggregation of work by many people. In online environments, interactivity can be viewed as a variable that characterizes the discussion, by representing the extent to which new posts are derived from the relations between existing posts [30]. This requires that participants engage with the contributions of their peers, and consider the already existing collective product in their own contributions. In the measurement of interactivity, we follow [21] in applying network measures to capture the interplay between participants and posts. We use the number of post views by peers, the ratio of reactive posts and the number of external links to establish the interactivity of the discussion:

Views. All 141 student participants viewed at least two posts by their peers. On average, each participant viewed 27 posts-by-peers, with the number for each participant ranging from 2 to 173 . The posts of 139 of the student participants $(99 \%)$ were viewed by their peers (at least one post by at least one peer). This suggests a very inclusive conversation, since practically all of the participants were "heard" by others, and the group as a whole was exposed to multiple perspectives.

Reactive posts. New posts could be either linked directly to one of the debate's opening posts or to a post by a peer. New posts that participants linked to posts by peers (other than themselves) were considered "reactive posts". 119 participants posted at least one such post, totaling 310 out of 673 posts (46\%). Additionally, 86 of the student participants received a reactive post from at least one fellow student ( $61 \%$ of all students). While contributing a reactive post was part of the graded assignment, nearly one-half of participating students posted more than one reaction to their peers.

External links. The platform lets participants link to external online resources, and they were encouraged to do so. Consequently, $43 \%$ of the posts included at least one external link, totaling 424 external links by students. $38 \%$ of the participants followed at least one of these links, adding another layer of interactivity.

The measures introduced here suggest nearly all of the participants interacted with content contributed by their peers. The network of content produced through this array of interactions is the product of collaboration, as each reactive post builds on its predecessors in the sequence. Collaboration offers participants an opportunity to generate collective knowledge which is greater than the mere aggregation of their inputs.

\subsection{Using emergent modules to identify emergent themes}

As explained above, the participants' sequential linking and cross-linking may result in the formation of distinct modules within the network of posts. These modules of more densely linked posts represent ideas or themes created collectively through the interactive discussion. Ideally, they should indicate that some convergence had occurred: ideas that weren't connected originally through the sequential development of the network could be bound together to form a novel combination. In terms of the network measures, this would be expressed in the reduction of the number of modules in the full graph, compared to a graph without the cross-links. The analysis, therefore, included detection of the modules, and then a comparison between the number of modules in the final graph to those found in the graph without the cross-links.

5.2.1 Extracting the modules. The modules were extracted using the walktrap.community method [31], which is based on random walks. The intuition behind it is that during a random walk along the edges of a graph, the likelihood of remaining within the same community is higher than moving across communities because the edges within the community are denser while ones bridging across communities are sparser [32]. Walktrap was selected for two reasons: first, it is considered relatively accurate and robust for small networks (<1000 nodes) [33]. Second, it complies with the idea of linking as a means of organizing modules within the collective conceptual map: the platform is built in a manner that encourages participants to navigate the network along the connections set by their peers, as traveling along existing direct links requires fewer actions. In this sense, the walktrap algorithm mimics the participants' navigation of the network, with an important difference being that the participants' navigation is not random but at least to some extent intentional, and based on the content of the posts.

The module extraction process was performed twice: for the full discussion graph and for an identical graph, with the cross-links removed. If the cross-links contribute to the formation of converged modules, representing new concepts or themes, then the graph with cross-links should contain fewer modules, as some of the prior modules converge into bigger ones through combination. The community detection algorithm was applied using the cluster_walktrap function from igraph [29]. This yielded 37 communities for the full, crosslinked graph, compared to 42 communities for the sequential, tree-shaped graph (12\% reduction). This implies that some convergence occurred, resulting in the emergence of new formations. 
5.2.2 Identifying emergent themes. Next, the modular network structure, which was formed based on the participants' links, was used for identifying themes formed through a collaborative combination process. We present the steps that were taken for extracting emergent ideas based on the input of participants on the connections between posts. We applied a combined process of network analysis supplemented by a qualitative phase.

The analysis consisted of the following steps:

1. Identifying the modules. The basis for identifying themes were the 37 modules extracted using the walktrap.community method (see above).

2. Recognizing the central themes. To recognize the main issue featured in each module, the post with the highest degree, i.e. the most connections to other posts, was singled out. This is because the most connected post acts as the epicenter of the module, and is the most related to issues raised in other posts, according to the participants. In cases where the main theme was not identifiable from the title, the post itself was read. For each module, some of the other posts were also examined, to give a more accurate description of the module theme and to make sure that the connections are coherent.

3. Drawing the graph. The module themes were graphically positioned within the graph structure, to display the general flow of the discussion. This was done by first laying the entire graph using forcedirected graph drawing techniques available in Gephi 0.9.2 [34]. These algorithms produce a graph display that incorporates the level of gravity between the nodes so that groups of nodes that are more densely connected will appear closer in the graphic display. The central node which initiates the entire discussion was manually removed from the module it was assigned to, as this was an artifact of the way the platform constructs the discussions, and irrelevant to the topical differentiation.

4. Creating captions. The titles for the leading posts in each module were used as the basis for creating captions. Where necessary, they were adapted to be more descriptive: some of the post titles were phrased as cultural references, questions, and other rhetorical means to attract attention. In some cases, the captions were modified to better describe the module based on several prominent posts.

5. Extracting the main flow. The graph was then filtered to include only the leading nodes within each module, without altering the nodes' positions. This resulted in a 37-node graph, which captures the main "skeleton" of the discussion. Each node representing a module was scaled in size in reference to the module's size (in terms of the number of nodes).

Figure 4 displays the community structure of the graph. Each color represents a different module.

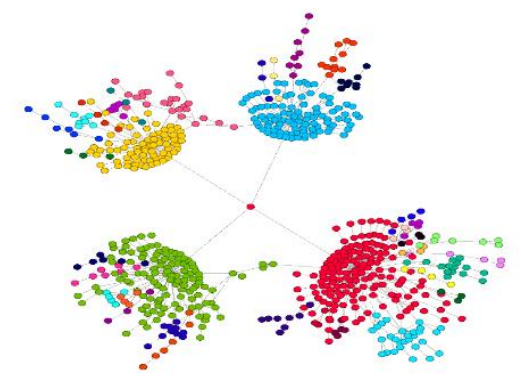

Figure 4. Full graph - modular structure

Figure 5 displays examples of the main themes emerging from the graph. See the full list of modules in the supplementary material.

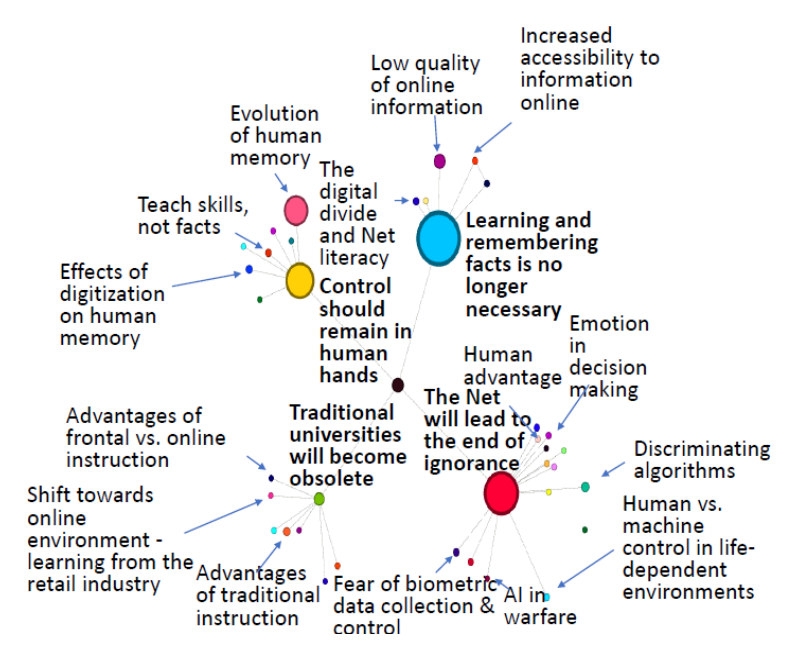

Figure 5. Some emergent themes

\subsection{Linking for new ideas: a qualitative example}

To demonstrate how a sequence of posting and linking evolves into insight, we zoom in on one fragment of the discussion and follow the posting and linking activities chronologically. The sequence begins with three separate posts, posted on days 14, 16 and 18 of the discussion, as replies to the proposition "Traditional universities will become obsolete". The posts are titled: (1) Not entirely true (universities will not disappear but they will have to change); (2) Lack of interaction and social isolation; (3) Compatibility between pedagogy and technology. On day 25, post (4) titled "Online learning: meaningful learning for anyone, anywhere" was posted. On day 27, posts (1) and (3) were linked. On day 30, post (5) titled "extroversion and 
introversion" was posted as a reply to post (2). On day 37, a post (6) titled "Academia is not suitable to the new world" was added. On day 39, posts (2) and (4) were linked. On day 42, a post (7) titled "Distant learning - $a$ miss-out on human interaction" was posted as a reply to post (4). On day 49, a post (8) titled "Frontal interaction is already diminishing" was posted in reply to post (2). On day 51, posts that were more integrative and more solution-oriented began appearing, starting with a post (9) titled "The future and future universities". Posts (9) and (2) were linked. On day 52 a post (10) titled "The future of education in a digital world" was posted and linked to post (9). This post suggests the application of the Self Organized Learning Environment model created by Sugata Mitra. The post also suggests that universities turn into a "one-stop-shop" which offers multiple formats of instruction and training, including short-term online courses. On the same day, another post (11) was added, titled "Higher education is living, breathing, kicking and more relevant than ever". It presents the crucial role of higher education institutes as mediators for learners in a changing world and suggests the growing practice of international collaborative academic study programs as a favorable direction for higher education institutions. Additionally, a post (12) titled "Their cheese was moved" was posted as a reply to post (1) and a new post (13) titled "Does online learning suit everyone?" was added. Post (12) was linked to post (1). On days 58 through 77, several cross-links among the posts appeared, creating a denser area within the graph and defining the scope of the segment. On day 77 Post (1) was linked to a post (14) from another sub-discussion: "Control must always remain in human hands". Post (14) was titled "Humans are not in competition with machines". It promoted educating for creative thinking and innovation, as opposed to systematic work that can be automatized.

The non-linear sequence described here, which included about a dozen more posts and many more external links, is representative of how a cross-linked interactive conversation supports emergence. From a bird's-eye view, it appears that this portion of the discussion was about recognizing the added values of traditional universities over online education, considering the advantages of online education, and combining these to identify directions that universities should follow to remain relevant in the near future. The more solution-orientated integrative posts began appearing almost simultaneously, around day 50 of the discussion. The link to the second sub-discussion, which appeared more than two months into the discussion, added another dimension to the conversation which is the role of higher education institutions as responsible for equipping future generations with relevant skills for a digitized world. The course of the deliberation was not dictated or promoted by instructors. It emerged spontaneously from the interactive conversation and was enabled by the platform's cross-linking feature.

\section{Discussion}

Online multi-participant collaborations can produce conversations which are quantitatively and qualitatively different than ones generated by co-located, size-limited and synchronic groups [35]. In this work, we examined the concept of collective creativity in online multiparticipant discussions. We presented interactivity and emergence as essential ingredients in collective creativity and discussed some of the affordances of online discussion platforms that enable and promote them. We highlighted the difference between sequential linking and cross-linking, and their various contributions to the topology of the discussion. By analyzing the network characteristics of a large-group discussion, we were able to demonstrate how these features contribute to both interaction and emergence within a specific discussion.

\subsection{Theoretical contribution}

The work presented here offers insight towards the mechanisms of producing new concepts through a largegroup online collaboration. It emphasizes the role of connection-making in the collaborative creative process: by making the connections public and available to the group, new meanings can emerge and the conversation advances. These connections act as organizing instruments, and they serve as a means for convergence via self-organization, without the need for external direction or top-down control. Their function gains significance in an era of distributed communities engaged in ongoing conversations and knowledge sharing. The research offers some preliminary indications for the potential role of self-organizing processes in facilitating the observation of creativity in distributed large groups collaborating online.

Although they were presented throughout this work as separate concepts, cross-linking can be viewed as a form of interactivity. It is another means of interacting with content, by further integrating it into context. It also enhances the interaction between the content units, because they become one another's contextual environments. Accordingly, the perception of interactivity in online conversations can be extended to include the forming and sharing of connections.

\subsection{Practical contribution}

This work turns the spotlight on the importance 
of cross-linking in online discussion platforms. Crosslinking breaks the linear topology dictated by a threaded discussion format and enables the rise of a networked structure, which is key for the emergence of new combinations. While different variations of this feature already exist in many platforms, our work suggests that they should be given even more weight.

The methodology presented here can be used in similar settings for extracting main themes out of a discussion, based on connections formed by participants explicitly. Methods for extracting themes using machine learning and text analysis require very large datasets for training and are limited to text-based similarities. Incorporating the semantic tagging input by participants regarding the connections between content units provides another direction for organizing multiparticipant discussions and identifying main themes, as well as the interplay among them. The process may be further developed for even more comprehensive and fine-tuned representations of the discussion's flow.

\section{Limitations and directions for future research}

The analysis was conducted on one discussion, held within the context of an academic course, within a specific platform. Future research should broaden the scope to include discussions from different contexts such as professional communities, public participation programs and more. It could be extended to different platforms, as long as they enable a cross-linking.

The participants were incentivized to be active in the discussion and to contribute to its organization for the good of the group. However, they were not explicitly asked to link or to react to peers' posts (aside from one mandatory reactive post). Initial findings from additional work on Ligilo data suggest that this behavior takes place even without any grade incentive, and crosslinking is also found in non-academic platforms [26]. Future work should further explore motivations for cross-linking in online discussions.

Creativity includes both generation and convergence phases. Fu and colleagues have addressed the issue of convergence based on concept-combination as a basis for subsequent idea generation in crowdsourcing environments [36]. The methodology that was applied here for module-formation based on cross-links can potentially be used for convergence: if the extraction of themes could be incorporated as a feature within the platform, rather than produced retroactively, it could serve as means to assist participants in grasping the developing "bigger picture". Convergent ideas could then perhaps be used as stepping stones for the generation of new ones, in an iterated process of convergence and divergence.

It should be noted that we presented the results of one community detection method. Some other methods that were explored yielded similar results, but others differ in resolution and in the modular structure produced. Future research should explore optimal methods for community detection, and perhaps add a qualitative evaluation of the modular structure.

On a more theoretical level, this work used the analogy between a collaborative discussion and the mental map of an individual as an inspiration. This direction could be further explored, by using current knowledge about individual creativity as reference for research on collective creativity in online environments.

\section{References}

[1] Haythornthwaite, C., Kumar, P., Gruzd, A., Gilbert, S., Esteve del Valle, M., and Paulin, D., "Learning in the wild: coding for learning and practice on Reddit", Learning, Media and Technology 43(3), 2018, pp. 219-235.

[2] Rice, R.E., Heinz, M., and van Zoonen, W., "A public goods model of outcomes from online knowledge sharing mediated by mental model processing", Journal of Knowledge Management 23(1), 2019, pp. 1-22.

[3] Sawyer, R.K., Explaining creativity: The science of human innovation, Oxford University Press, 2012.

[4] Cranshaw, J., and Kittur, A., "The Polymath Project: Lessons from a Successful Online Collaboration in Mathematics", Proceedings of the 2011 Annual Conference on Computer Human Interaction, 2011, pp. 1865-1874.

[5] Yu, L., Nickerson, J. V., and Sakamoto, Y., "Collective Creativity: Where we are and where we might go", Proceedings of collective intelligence., (2012).

[6] Kent, C., and Rafaeli, S., "How interactive is a semantic network? concept maps and discourse in knowledge communities", Proceedings of the Annual Hawaii International Conference on System Sciences, (2016).

[7] Heylighen, F., Heath, M., and Van, F., "The Emergence of Distributed Cognition: a conceptual framework", Proceedings of collective intentionality IV IV(January), 2004, pp. 20.

[8] Sarmiento, J.W., and Stahl, G., "Group creativity in interaction: Collaborative referencing, remembering, and bridging", International Journal of Human-Computer Interaction 24(5), 2008, pp. 492-504.

[9] Paulus, P.B., and Yang, H.C., "Idea generation in groups: A basis for creativity in organizations", Organizational Behavior and Human Decision Processes 82(1), 2000, pp. 7687. 
[10] Rafaeli, S., and Sudweeks, F., "Networked Interactivity", Journal of Computer-Mediated Communication 2(4), 1997, pp. JCMC243.

[11] Sawyer, R.K., John-Steiner, V., Moran, S., et al., Creativity and Development, 2012.

[12] Johnson, S., Emergence: The Connected Lives of Ants, Brains, Cities, and Software, Scribner, New York, 2001.

[13] Schilling, M.A., "A 'small-world' network model of cognitive insight", Creativity Research Journal 17(2-3), 2005, pp. 131-154.

[14] Kenett, Y.N., Anaki, D., and Faust, M., "Investigating the structure of semantic networks in low and high creative persons", Frontiers in Human Neuroscience 8(June), 2014, pp. 1-16.

[15] Stahl, G., Group Cognition Computer Support for Building Collaborative Knowledge, MIT Press, Cambridge, Massachusetts, 2006.

[16] Stahl, G., "Group cognition in computer-assisted collaborative learning", Journal of Computer Assisted Learning 21(2), 2005, pp. 79-90.

[17] Zhang, A.X., Verou, L., and Karger, D., "Wikum: Bridging Discussion Forums and Wikis Using Recursive Summarization", Proceedings of the 2017 ACM Conference on Computer Supported Cooperative Work and Social Computing - CSCW'17, (2017).

[18] Hoque, E., and Carenini, G., "ConVisiIT: Interactive Topic Modeling for Exploring Asynchronous Online Conversations", ACM Transactions on Interactive Intelligent Systems 6(1), 2016, pp. 1-24.

[19] Chan, J., Chang, J.C., Hope, T., Shahaf, D., and Kittur, A., "SOLVENT: A Mixed Initiative System for Finding Analogies between Research Papers", Proceedings of the ACM on Human-Computer Interaction, (2018).

[20] Kent, C., Rechavi, A., and Rafaeli, S., Networked Learning Analytics: A Theoretically Informed Methodology for Analytics of Collaborative Learning, Springer, Cham, 2019.

[21] Kent, C., and Rafaeli, S., "How interactive is a semantic network? concept maps and discourse in knowledge communities", Proceedings of the Annual Hawaii International Conference on System Sciences 2016-March, 2016, pp. 2095-2104.

[22] Siemens, G., Knowing Knowledge, Lulu.com. A Creative Commons License., 2006.

[23] Biasutti, M., "A comparative analysis of forums and wikis as tools for online collaborative learning", Computers and Education 111, 2017, pp. 158-171.

[24] Salter, N.P., and Conneely, M.R., "Structured and unstructured discussion forums as tools for student engagement", Computers in Human Behavior 46, 2015, pp. $18-25$.

[25] Hewitt, J., "Beyond threaded discourse", International Journal of Educational Telecommunications 7, 2001, pp. 207221.

[26] Ye, D., Xing, Z., and Kapre, N., "The structure and dynamics of knowledge network in domain-specific Q\&A sites: a case study of stack overflow", Empirical Software Engineering 22(1), 2017, pp. 375-406.

[27] Houghton, J.P., Siegel, M., Madnick, S., et al., "Beyond Keywords: Tracking the Evolution of Conversational Clusters in Social Media", Sociological Methods and Research(October), 2017.

[28] Kimmerle, J., Moskaliuk, J., Harrer, A., and Cress, U., "Visualizing co-evolution of individual and collective knowledge", Information, Communication \& Society 13(8), 2010, pp. 1099-1121.

[29] Csardi, G., and Nepusz, T., "The igraph software package for complex network research", InterJournal, Complex Systems 1695, 2006, pp. 1-9.

[30] Rafaeli, S., "Interactivity: From new media to communication.", In R.P. Hawkins, J.M. Wieman and S. Pingree, eds., Advancing communication science: Merging mass and interpersonal processes. Sage, Newbury Park, CA, 1988, 110-134.

[31] Pons, P., and Latapy, M., "Computing communities in large networks using random walks", International symposium on computer and information sciences 10(2), 2005, pp. 1-20.

[32] Jalal, M., and Doan, A., A Survey on Community Detection Methods in Social Networks, 2015.

[33] Yang, Z., Algesheimer, R., and Tessone, C.J., "Corrigendum: A Comparative Analysis of Community Detection Algorithms on Artificial Networks", Scientific reports 7(August), 2017, pp. 46845.

[34] Bastian, M., Heymann, S., and Jacomy, M., "Gephi: An Open Source Software for Exploring and Manipulating Networks", International AAAI Conference on Weblogs and Social Media, (2009).

[35] Erickson, T., "Persistent Conversation: an Introduction", Journal of Computer-Mediated Communication 4(4, 1 June), 1999.

[36] Fu, S., Cheng, X., de Vreede, T., et al., "Exploring Idea Convergence and Conceptual Combination in Open Innovative Crowdsourcing from a Cognitive Load Perspective", Proceedings of the 52nd Hawaii International Conference on System Sciences, 2019, pp. 315-324. 